Supplement of Biogeosciences, 14, 3603-3613, 2017

https://doi.org/10.5194/bg-14-3603-2017-supplement

(C) Author(s) 2017. This work is distributed under

the Creative Commons Attribution 3.0 License.

(c) (1)

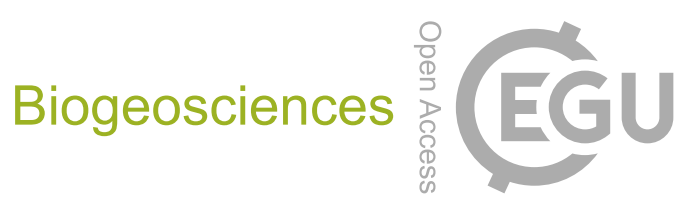

Supplement of

\title{
Impact of trace metal concentrations on coccolithophore growth and morphology: laboratory simulations of Cretaceous stress
}

Giulia Faucher et al.

Correspondence to: Giulia Faucher (giulia.faucher@unimi.it)

The copyright of individual parts of the supplement might differ from the CC BY 3.0 License. 


\begin{tabular}{|c|c|c|c|c|}
\hline \multicolumn{5}{|c|}{ Growth rate } \\
\hline & Control & Low & Medium & High \\
\hline r1 & 0.54 & 0.57 & 0.56 & 0.57 \\
r2 & 0.50 & 0.55 & 0.56 & 0.56 \\
r3 & 0.52 & 0.58 & 0.56 & 0.56 \\
\hline Average & 0.52 & 0.57 & 0.56 & 0.57 \\
\hline
\end{tabular}

$r=$ replicate

\begin{tabular}{|c|c|c|c|c|}
\hline \multicolumn{5}{|c|}{ Coccosphere Diameter } \\
\hline & Control & Low & Medium & High \\
\hline r1 & 11.80 & 12.17 & 11.89 & 12.07 \\
r2 & 11.59 & 12.01 & 11.86 & 12.09 \\
r3 & 11.72 & 12.15 & 11.88 & 11.81 \\
\hline Average & 11.70 & 12.11 & 11.88 & 11.99 \\
\hline
\end{tabular}

\begin{tabular}{|c|c|c|c|c|}
\hline \multicolumn{5}{|c|}{ Cell Diameter } \\
\hline r1 & Control & Low & Medium & High \\
r2 & 9.36 & 9.01 & 8.97 & 9.01 \\
r3 & 8.71 & 8.82 & 9.00 & 8.98 \\
\hline Average & 9.02 & 8.96 & 9.09 & 8.95 \\
\hline
\end{tabular}

\begin{tabular}{|c|c|c|c|c|}
\hline \multicolumn{5}{|c|}{ VCP } \\
\hline r1 & Control & Low & Medium & High \\
r2 & 440.16 & 577.15 & 507.49 & 555.45 \\
r3 & 473.89 & 538.06 & 501.00 & 551.27 \\
\hline Average & 475.14 & 595.43 & 494.41 & 492.81 \\
\hline
\end{tabular}

Tab.S1 Growth rate $\left(\mathrm{d}^{-1}\right)$, coccosphere and cell diameters $(\mu \mathrm{m})$ and volume of the calcitic part $\left(\mathrm{VCP} ; \mu \mathrm{m}^{3}\right)$ of $E$. huxleyi at the end of our experiments for each replicate. 


\begin{tabular}{|c|c|c|c|c|}
\hline \multicolumn{5}{|c|}{ Growth rate } \\
\hline & Control & Low & Medium & High \\
\hline r1 & 0.66 & 0.58 & 0.59 & 0.56 \\
r2 & 0.66 & 0.58 & 0.60 & 0.59 \\
r3 & 0.67 & 0.58 & 0.62 & 0.59 \\
\hline Average & 0.66 & 0.58 & 0.60 & 0.58 \\
\hline
\end{tabular}

$\mathrm{r}=$ replicate

\begin{tabular}{|c|c|c|c|c|}
\multicolumn{6}{|c|}{ Coccosphere Diameter } \\
\hline & Control & Low & Medium & High \\
\hline r1 & 7.25 & 6.60 & 6.58 & 6.09 \\
r2 & 7.26 & 6.58 & 6.55 & 6.17 \\
r3 & 7.24 & 6.56 & 6.67 & 6.16 \\
\hline Average & 7.25 & 6.58 & 6.60 & 6.14 \\
\hline
\end{tabular}

\begin{tabular}{|c|c|c|c|c|}
\hline \multicolumn{5}{|c|}{ Cell Diameter } \\
\hline & Control & Low & Medium & High \\
\hline r1 & 5.42 & 5.16 & 5.17 & 4.72 \\
r3 & 5.51 & 5.10 & 5.16 & 4.78 \\
\hline Average & 5.41 & 5.29 & 5.23 & 4.74 \\
\hline
\end{tabular}

\begin{tabular}{|c|c|c|c|c|}
\hline \multicolumn{5}{|c|}{ VCP } \\
\hline r1 & Control & Low & Medium & High \\
r2 & 116.84 & 77.42 & 75.59 & 60.61 \\
r3 & 91.13 & 76.79 & 74.21 & 63.39 \\
\hline Average & 95.08 & 72.24 & 82.43 & 64.89 \\
\hline
\end{tabular}

Tab.S2 Growth rate $\left(\mathrm{d}^{-1}\right)$, coccosphere and cell diameters $(\mu \mathrm{m})$ and volume of the calcitic part $\left(\mathrm{VCP} ; \mu \mathrm{m}^{3}\right)$ of G. oceanica at the end of our experiments for each replicate. 


\begin{tabular}{|c|c|c|c|c|}
\hline \multicolumn{5}{|c|}{ Growth rate } \\
\hline & Control & Low & Medium & High \\
\hline r1 & 0.55 & 0.42 & 0.47 & 0.44 \\
r2 & 0.56 & 0.43 & 0.42 & 0.44 \\
r3 & 0.56 & 0.42 & 0.39 & 0.42 \\
\hline Average & 0.56 & 0.42 & 0.43 & 0.43 \\
\hline
\end{tabular}

$r=$ replicate

\begin{tabular}{|c|c|c|c|c|}
\multicolumn{5}{|c|}{ Coccosphere Diameter } \\
\hline & Control & Low & Medium & High \\
\hline r1 & 19.70 & 17.01 & 16.96 & 16.98 \\
r2 & 19.90 & 17.21 & 16.91 & 17.13 \\
r3 & 19.85 & 17.15 & 17.27 & 16.45 \\
\hline Average & 19.82 & 17.12 & 17.05 & 16.85 \\
\hline
\end{tabular}

\begin{tabular}{|c|c|c|c|c|}
\hline \multicolumn{5}{|c|}{ Cell Diameter } \\
\hline & Control & Low & Medium & High \\
\hline r1 & 14.05 & 10.09 & 10.16 & 10.35 \\
r2 & 16.57 & 10.13 & 10.11 & 10.34 \\
r3 & 16.33 & 10.09 & 11.10 & 10.45 \\
\hline Average & 15.65 & 10.10 & 10.46 & 10.38 \\
\hline
\end{tabular}

\begin{tabular}{|c|c|c|c|c|}
\hline \multicolumn{5}{|c|}{ VCP } \\
\hline r1 & Control & Low & Medium & High \\
r2 & 1751.16 & 2112.43 & 2071.18 & 1919.11 \\
r3 & 1806.95 & 2133.68 & 1966.05 & 2153.53 \\
\hline Average & 1722.13 & 2062.28 & 2072.10 & 1789.59 \\
\hline
\end{tabular}

Tab.S3 Growth rate $\left(\mathrm{d}^{-1}\right)$, coccosphere and cell diameters $(\mu \mathrm{m})$ and volume of the calcitic part (VCP; $\left.\mu \mathrm{m}^{3}\right)$ of C. pelagicus at the end of our experiments for each replicate. 


\begin{tabular}{|c|c|c|c|c|}
\hline \multicolumn{5}{|c|}{ Growth rate } \\
\hline & Control & Low & Medium & High \\
\hline r1 & 0.54 & 0.57 & 0.56 & 0.57 \\
r2 & 0.50 & 0.55 & 0.56 & 0.56 \\
r3 & 0.52 & 0.58 & 0.56 & 0.56 \\
\hline Average & 0.52 & 0.57 & 0.56 & 0.57 \\
\hline
\end{tabular}

$r=$ replicate

\begin{tabular}{|c|c|c|c|c|}
\hline \multicolumn{5}{|c|}{ Coccosphere Diameter } \\
\hline & Control & Low & Medium & High \\
\hline r1 & 11.80 & 12.17 & 11.89 & 12.07 \\
r2 & 11.59 & 12.01 & 11.86 & 12.09 \\
r3 & 11.72 & 12.15 & 11.88 & 11.81 \\
\hline Average & 11.70 & 12.11 & 11.88 & 11.99 \\
\hline
\end{tabular}

\begin{tabular}{|c|c|c|c|c|}
\hline \multicolumn{5}{|c|}{ Cell Diameter } \\
\hline & Control & Low & Medium & High \\
\hline r1 & 9.36 & 9.01 & 8.97 & 9.01 \\
r2 & 8.71 & 8.82 & 9.00 & 8.98 \\
r3 & 9.02 & 8.96 & 9.09 & 8.95 \\
\hline Average & 9.03 & 8.93 & 9.02 & 8.98 \\
\hline
\end{tabular}

\begin{tabular}{|c|c|c|c|c|}
\hline \multicolumn{5}{|c|}{ VCP } \\
\hline r1 & Control & Low & Medium & High \\
r2 & 440.16 & 577.15 & 507.49 & 555.45 \\
r3 & 473.89 & 538.06 & 501.00 & 551.27 \\
\hline Average & 475.14 & 595.43 & 494.41 & 492.81 \\
\hline
\end{tabular}

Tab.S4 Growth rate $\left(\mathrm{d}^{-1}\right)$, coccosphere and cell diameters $(\mu \mathrm{m})$ and volume of the calcitic part $\left(\mathrm{VCP} ; \mu \mathrm{m}^{3}\right)$ of $P$. carterae at the end of our experiments for each replicate. 
E. huxleyi

\begin{tabular}{|c|c|c|c|c|c|c|}
\hline \multirow{6}{*}{$\begin{array}{l}\text { LOW } \\
\text { MEAN } \\
\text { MIN } \\
\text { MAX } \\
\text { MEDIAN }\end{array}$} & \multicolumn{2}{|c|}{ Replicate 1} & \multicolumn{2}{|c|}{ Replicate 2} & \multicolumn{2}{|c|}{ Replicate 3} \\
\hline & DSL & DSW & DSL & DSW & DSL & DSW \\
\hline & 2.67 & 2.14 & 2.65 & 2.09 & 2.66 & 2.10 \\
\hline & 2.12 & 1.73 & 2.15 & 1.65 & 1.98 & 1.43 \\
\hline & 3.17 & 2.64 & 3.27 & 2.70 & 3.36 & 2.81 \\
\hline & 2.67 & 2.09 & 2.65 & 2.09 & 2.67 & 2.08 \\
\hline \multirow{6}{*}{$\begin{array}{l}\text { MEDIUM } \\
\text { MEAN } \\
\text { MIN } \\
\text { MAX } \\
\text { MEDIAN }\end{array}$} & \multicolumn{2}{|c|}{ Replicate 1} & \multicolumn{2}{|c|}{ Replicate 2} & \multicolumn{2}{|c|}{ Replicate 3} \\
\hline & $\overline{D S L}$ & DSW & $\overline{\mathrm{DSL}}$ & DSW & $\overline{\mathrm{DSL}}$ & DSW \\
\hline & 2.68 & 2.11 & 2.62 & 2.06 & 2.63 & 2.02 \\
\hline & 1.93 & 1.40 & 2.00 & 1.52 & 2.09 & 1.21 \\
\hline & 3.61 & 3.01 & 3.19 & 2.61 & 4.52 & 3.44 \\
\hline & 2.66 & 2.06 & 2.63 & 2.09 & 2.60 & 1.98 \\
\hline \multirow{6}{*}{$\begin{array}{c}\text { MAX } \\
\text { MEAN } \\
\text { MIN } \\
\text { MAX } \\
\text { MEDIAN }\end{array}$} & \multicolumn{2}{|c|}{ Replicate 1} & \multicolumn{2}{|c|}{ Replicate 2} & \multicolumn{2}{|c|}{ Replicate 3} \\
\hline & $\mathrm{DSL}$ & DSW & $\overline{D S L}$ & DSW & $\mathrm{DSL}$ & DSW \\
\hline & 2.58 & 1.97 & 2.58 & 1.99 & 2.61 & 1.99 \\
\hline & 1.78 & 1.44 & 2.04 & 1.60 & 1.55 & 1.06 \\
\hline & 3.35 & 2.59 & 3.25 & 2.61 & 3.22 & 2.67 \\
\hline & 2.57 & 1.95 & 2.57 & 1.98 & 2.66 & 2.01 \\
\hline \multirow{6}{*}{$\begin{array}{c}\text { CONTROL } \\
\text { MEAN } \\
\text { MIN } \\
\text { MAX } \\
\text { MEDIAN }\end{array}$} & \multicolumn{2}{|c|}{ Replicate 1} & \multicolumn{2}{|c|}{ Replicate 2} & \multicolumn{2}{|c|}{ Replicate 3} \\
\hline & $\mathrm{DSL}$ & DSW & $\overline{D S L}$ & DSW & $\overline{D S L}$ & DSW \\
\hline & 2.93 & 2.38 & 3.04 & 2.49 & 2.97 & 2.44 \\
\hline & 2.34 & 1.83 & 2.38 & 1.95 & 2.09 & 1.75 \\
\hline & 3.65 & 3.14 & 3.69 & 3.06 & 3.86 & 3.45 \\
\hline & 2.95 & 2.39 & 3.04 & 2.46 & 2.94 & 2.40 \\
\hline
\end{tabular}

G. oceanica

\begin{tabular}{|c|c|c|c|c|c|c|}
\hline \multirow{6}{*}{$\begin{array}{c}\text { LOW } \\
\text { MEAN } \\
\text { MIN } \\
\text { MAX } \\
\text { MEDIAN }\end{array}$} & \multicolumn{2}{|c|}{ Replicate 1} & \multicolumn{2}{|c|}{ Replicate 2} & \multicolumn{2}{|c|}{ Replicate 3} \\
\hline & $\mathrm{DSL}$ & DSW & DSL & DSW & $\mathrm{DSL}$ & DSW \\
\hline & 4.02 & 3.46 & 4.12 & 3.61 & 4.15 & 3.63 \\
\hline & 3.06 & 2.47 & 3.30 & 2.70 & 3.27 & 2.79 \\
\hline & 4.84 & 4.55 & 5.30 & 4.90 & 5.38 & 4.95 \\
\hline & 4.00 & 3.46 & 4.04 & 3.51 & 4.14 & 3.61 \\
\hline \multirow{6}{*}{$\begin{array}{l}\text { MEDIUM } \\
\text { MEAN } \\
\text { MIN } \\
\text { MAX } \\
\text { MEDIAN }\end{array}$} & \multicolumn{2}{|c|}{ Replicate 1} & \multicolumn{2}{|c|}{ Replicate 2} & \multicolumn{2}{|c|}{ Replicate 3} \\
\hline & $\overline{\mathrm{DSL}}$ & DSW & $\overline{\mathrm{DSL}}$ & DSW & $\overline{D S L}$ & DSW \\
\hline & 4.16 & 3.56 & 4.18 & 3.60 & 4.13 & 3.59 \\
\hline & 3.12 & 2.58 & 3.22 & 2.53 & 3.19 & 2.77 \\
\hline & 5.15 & 4.47 & 5.89 & 5.15 & 5.70 & 4.78 \\
\hline & 4.17 & 3.51 & 4.14 & 3.55 & 4.06 & 3.48 \\
\hline \multirow{6}{*}{$\begin{array}{c}\text { MAX } \\
\text { MEAN } \\
\text { MIN } \\
\text { MAX } \\
\text { MEDIAN }\end{array}$} & \multicolumn{2}{|c|}{ Replicate 1} & \multicolumn{2}{|c|}{ Replicate 2} & \multicolumn{2}{|c|}{ Replicate 3} \\
\hline & $\overline{D S L}$ & DSW & $\overline{\mathrm{DSL}}$ & DSW & DSL & DSW \\
\hline & 3.96 & 3.34 & 3.89 & 3.30 & 4.06 & 3.47 \\
\hline & 3.13 & 2.57 & 3.18 & 2.47 & 3.34 & 2.80 \\
\hline & 5.09 & 4.75 & 5.32 & 4.64 & 5.73 & 5.21 \\
\hline & 3.96 & 3.29 & 3.77 & 3.19 & 4.05 & 3.42 \\
\hline \multirow{6}{*}{$\begin{array}{c}\text { CONTROL } \\
\text { MEAN } \\
\text { MIN } \\
\text { MAX } \\
\text { MEDIAN }\end{array}$} & \multicolumn{2}{|c|}{ Replicate 1} & \multicolumn{2}{|c|}{ Replicate 2} & \multicolumn{2}{|c|}{ Replicate 3} \\
\hline & $\overline{\mathrm{DSL}}$ & DSW & $\overline{\mathrm{DSL}}$ & DSW & $\overline{\mathrm{DSL}}$ & DSW \\
\hline & 4.26 & 3.72 & 4.26 & 3.73 & 4.42 & 3.87 \\
\hline & 3.28 & 2.93 & 3.24 & 2.90 & 3.41 & 3.11 \\
\hline & 5.18 & 4.41 & 5.11 & 4.45 & 5.78 & 4.77 \\
\hline & 4.25 & 3.76 & 4.27 & 3.73 & 4.37 & 3.85 \\
\hline
\end{tabular}

Table S5. E. huxleyi and G. oceanica coccolith distal shield length (DSL) and distal shield width (DSW): mean, minimum (MIN), maximum (MAX) and median values ( $n=50$ coccoliths measurements for each replicate) for every treatment, at the end of the experiment. 
C. pelagicus

\begin{tabular}{|c|c|c|c|c|c|c|}
\hline \multirow{6}{*}{$\begin{array}{c}\text { LOW } \\
\text { MEAN } \\
\text { MIN } \\
\text { MAX } \\
\text { MEDIAN }\end{array}$} & \multicolumn{2}{|c|}{ Replicate 1} & \multicolumn{2}{|c|}{ Replicate 2} & \multicolumn{2}{|c|}{ Replicate 3} \\
\hline & $\mathrm{DSL}$ & DSW & DSL & DSW & DSL & DSW \\
\hline & 10.48 & 8.55 & 10.31 & 8.25 & 10.52 & 8.50 \\
\hline & 7.99 & 6.15 & 7.18 & 5.61 & 8.67 & 6.82 \\
\hline & 12.50 & 10.35 & 12.65 & 10.20 & 11.84 & 9.78 \\
\hline & 10.53 & 8.61 & 10.47 & 8.38 & 10.60 & 8.62 \\
\hline \multirow{6}{*}{$\begin{array}{l}\text { MEDIUM } \\
\text { MEAN } \\
\text { MIN } \\
\text { MAX } \\
\text { MEDIAN } \\
\end{array}$} & \multicolumn{2}{|c|}{ Replicate 1} & \multicolumn{2}{|c|}{ Replicate 2} & \multicolumn{2}{|c|}{ Replicate 3} \\
\hline & $\mathrm{DSL}$ & DSW & $\mathrm{DSL}$ & DSW & DSL & DSW \\
\hline & 10.17 & 8.28 & 10.04 & 8.16 & 9.88 & 8.07 \\
\hline & 8.65 & 6.68 & 7.38 & 5.59 & 6.99 & 5.62 \\
\hline & 11.65 & 9.57 & 12.68 & 10.05 & 12.21 & 9.68 \\
\hline & 10.29 & 8.18 & 10.05 & 8.23 & 9.91 & 8.12 \\
\hline \multirow{6}{*}{$\begin{array}{c}\text { MAX } \\
\text { MEAN } \\
\text { MIN } \\
\text { MAX } \\
\text { MEDIAN }\end{array}$} & \multicolumn{2}{|c|}{ Replicate 1} & \multicolumn{2}{|c|}{ Replicate 2} & \multicolumn{2}{|c|}{ Replicate 3} \\
\hline & $\mathrm{DSL}$ & DSW & $\mathrm{DSL}$ & DSW & $\mathrm{DSL}$ & DSW \\
\hline & 10.70 & 8.63 & 10.33 & 8.40 & 10.37 & 8.47 \\
\hline & 9.14 & 6.98 & 8.35 & 6.56 & 8.47 & 6.95 \\
\hline & 12.16 & 10.25 & 12.66 & 10.65 & 12.63 & 10.60 \\
\hline & 10.57 & 8.60 & 10.30 & 8.40 & 10.43 & 8.60 \\
\hline \multirow{6}{*}{$\begin{array}{l}\text { CONTROL } \\
\text { MEAN } \\
\text { MIN } \\
\text { MAX } \\
\text { MEDIAN }\end{array}$} & \multicolumn{2}{|c|}{ Replicate 1} & \multicolumn{2}{|c|}{ Replicate 2} & \multicolumn{2}{|c|}{ Replicate 3} \\
\hline & $\mathrm{DSL}$ & DSW & DSL & DSW & $\mathrm{DSL}$ & DSW \\
\hline & 12.99 & 11.33 & 12.52 & 10.91 & 12.78 & 11.00 \\
\hline & 11.43 & 10.00 & 11.01 & 10.00 & 11.15 & 10.08 \\
\hline & 14.85 & 13.86 & 14.66 & 12.80 & 14.15 & 11.62 \\
\hline & 12.83 & 11.11 & 12.43 & 10.69 & 12.76 & 10.94 \\
\hline
\end{tabular}

P. carterae

\begin{tabular}{|c|c|c|c|c|c|c|}
\hline \multirow{6}{*}{$\begin{array}{l}\text { LOW } \\
\text { MEAN } \\
\text { MIN } \\
\text { MAX } \\
\text { MEDIAN }\end{array}$} & \multicolumn{2}{|c|}{ Replicate 1} & \multicolumn{2}{|c|}{ Replicate 2} & \multicolumn{2}{|c|}{ Replicate 3} \\
\hline & DSL & DSW & DSL & DSW & $\mathrm{DSL}$ & DSW \\
\hline & 1.90 & 1.17 & 1.86 & 1.16 & 1.93 & 1.21 \\
\hline & 2.29 & 1.37 & 2.18 & 1.42 & 2.24 & 1.46 \\
\hline & 2.29 & 1.37 & 2.18 & 1.42 & 2.24 & 1.46 \\
\hline & 1.87 & 1.18 & 1.87 & 1.17 & 1.94 & 1.20 \\
\hline \multirow{6}{*}{$\begin{array}{l}\text { MEDIUM } \\
\text { MEAN } \\
\text { MIN } \\
\text { MAX } \\
\text { MEDIAN }\end{array}$} & \multicolumn{2}{|c|}{ Replicate 1} & \multicolumn{2}{|c|}{ Replicate 2} & \multicolumn{2}{|c|}{ Replicate 3} \\
\hline & $\mathrm{DSL}$ & DSW & $\mathrm{DSL}$ & DSW & $\mathrm{DSL}$ & DSW \\
\hline & 1.86 & 1.13 & 1.87 & 1.17 & 1.87 & 1.18 \\
\hline & 1.65 & 0.89 & 1.56 & 0.98 & 1.61 & 0.97 \\
\hline & 2.11 & 1.27 & 2.16 & 1.49 & 2.15 & 1.53 \\
\hline & 1.87 & 1.14 & 1.86 & 1.15 & 1.86 & 1.17 \\
\hline \multirow{2}{*}{ MAX } & \multicolumn{2}{|c|}{ Replicate 1} & \multicolumn{2}{|c|}{ Replicate 2} & \multicolumn{2}{|c|}{ Replicate 3} \\
\hline & DSL & DSW & DSL & DSW & $\mathrm{DSL}$ & DSW \\
\hline \multirow{4}{*}{$\begin{array}{l}\text { MEAN } \\
\text { MIN } \\
\text { MAX } \\
\text { MEDIAN }\end{array}$} & 1.90 & 1.18 & 1.91 & 1.19 & 1.90 & 1.19 \\
\hline & 1.59 & 1.00 & 1.62 & 1.01 & 1.62 & 1.02 \\
\hline & 2.26 & 1.53 & 2.12 & 1.43 & 2.16 & 1.38 \\
\hline & 1.89 & 1.18 & 1.91 & 1.18 & 1.90 & 1.20 \\
\hline \multirow{6}{*}{$\begin{array}{l}\text { CONTROL } \\
\text { MEAN } \\
\text { MIN } \\
\text { MAX } \\
\text { MEDIAN }\end{array}$} & \multicolumn{2}{|c|}{ Replicate 1} & \multicolumn{2}{|c|}{ Replicate 2} & \multicolumn{2}{|c|}{ Replicate 3} \\
\hline & DSL & DSW & DSL & DSW & $\mathrm{DSL}$ & DSW \\
\hline & 1.90 & 1.17 & 1.86 & 1.16 & 1.93 & 1.21 \\
\hline & 1.59 & 0.95 & 1.38 & 0.96 & 1.67 & 0.99 \\
\hline & 2.29 & 1.37 & 2.18 & 1.42 & 2.24 & 1.46 \\
\hline & 1.87 & 1.18 & 1.87 & 1.17 & 1.94 & 1.20 \\
\hline
\end{tabular}

Table S6. C. pelagicus and $P$. carterae coccolith distal shield length (DSL) and distal shield width (DSW): mean, minimum (MIN), maximum (MAX) and median values $(n=50$ coccoliths measurements for each replicate) for every treatment, at the end of the experiment 